\title{
Educación en ciencias desde diferentes contextos culturales y ambientales: contribuciones didácticas curriculares
}

\author{
Sandra Elvira Ruiz Castillo ${ }^{1}{ }^{10}$ \\ Universidad Distrital Francisco José de Caldas, Colombia
}

Autor de correspondencia:

1'draizgec@gmial.com

Recibido: 01 de junio de 2020

Revisado: 28 de julio de 2020

Aprobado: 30 de diciembre de 2020

Publicado: 06 de marzo de 2021

\section{Resumen}

En este artículo se evidencia la necesidad de vincular la educación de ciencias con la diversidad cultural y ambiental, para la construcción pedagógica y didáctica de la ciencia escolar, con el fin de integrar el mundo de la vida con las interacciones que en él se generan para la construcción de conocimiento. Los objetivos fueron hallar el sentido que le otorgan profesores de básica y media a la vinculación del contexto cultural y ambiental en la educación en ciencias; y analizar sus ideas y experiencias significativas. La metodología tuvo un carácter cualitativo que, en lo ontológico, abordó una realidad de naturaleza cultural diversa y subjetiva; y un carácter hermenéutico, por el tipo de análisis interpretativo de los datos. En los resultados, se destaca la construcción de categorías para integrar el contexto cultural y ambiental en las construcciones didácticas para promover aprendizajes en los que se reconocen la ciencia como construcción cultural y los aportes para la transformación curricular al integrar características de los territorios.

Palabras clave: diversidad cultural, diversidad ambiental, educación en ciencias

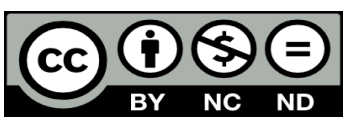

Para citar este artículo: Ruiz, S. (2021). Educación en ciencias desde diferentes contextos culturales y ambientales: contribuciones didácticas curriculares. Praxis \& Saber, 12(31), e11101. https://doi.org/10.19053/22160159.v12.n31.2021.11101 


\title{
Science education from different cultural and environmental contexts: didactic contributions to the curriculum
}

\begin{abstract}
This article emphasizes the need to connect science education with cultural and environmental diversity, for the pedagogical and didactic construction of school science, in order to integrate the world of life with the interactions generated in it for the construction of knowledge. The objectives were to find the meanings given by elementary and middle school teachers to the connection between the cultural and environmental context in science education; and to analyze their ideas and meaningful experiences. The methodology had a qualitative aspect which, on the ontological side, approached a culturally diverse and subjective reality; and a hermeneutic aspect, due to the type of interpretative analysis of data. In the results, it is highlighted the construction of categories to integrate the cultural and environmental context in the didactic constructs to promote learning, in which both science as a cultural construction and the contributions for the curricular transformation when integrating characteristics of the territory are recognized.
\end{abstract}

Keywords: cultural diversity, environmental diversity, science education

\section{Ensino de ciências em diferentes contextos culturais e ambientais: contribuições didáticas e curriculares}

\section{Resumo}

Este artigo apresenta a necessidade de ligar a educação científica com a diversidade cultural e ambiental, para a construção pedagógica e didática da ciência escolar, a fim de integrar o mundo da vida com as interações nele geradas para a construção do conhecimento. Os objetivos foram encontrar o sentido dado pelos professores do ensino fundamental e médio à vinculação do contexto cultural e ambiental na educação científica; e analisar suas ideias e experiências significativas. A metodologia teve um caráter qualitativo que, no aspecto ontológico, abordou uma realidade de natureza cultural diversa e subjetiva; e um caráter hermenêutico, por causa do tipo de análise interpretativa dos dados. Nos resultados, destaca-se a construção de categorias para integrar o contexto cultural e ambiental nas construções didáticas para promover a aprendizagem na qual se reconhece a ciência como uma construção cultural e as contribuições para a transformação curricular através da integração das características dos territórios.

Palavras-chave: diversidade cultural, diversidade ambiental, ensino de ciências

Este artículo es producto de la investigación desarrollada por la autora en el marco del Doctorado en Educación, desarrollado en la Universidad Distrital Francisco José de Caldas. El texto es pertinente en la medida que atiende a necesidades y expectativas de 
la sociedad frente a la educación de los niños, niñas y jóvenes en ciencias, a propósito de comprender si la ciencia escolar se concibe, desde el profesorado de educación básica y media, como una construcción cultural en permanente evolución que presenta diferentes niveles de incidencia, tanto en la subjetividad de cada individuo como en las interacciones colectivas y su contexto cultural y ambiental. Esta postura genera el reto de aprender a leer el mundo para que la educación de las ciencias trascienda a partir de criterios pedagógicos y didácticos que dinamicen la construcción de la ciencia escolar y permitan configurar otras formas de interactuar, al reconocer tanto la diversidad cultural y ambiental como los fines comunes que buscan la convivencia, la sostenibilidad y el desarrollo, "para que en las aulas culturalmente sensibles el profesorado aprenda de los estudiantes, los estudiantes aprendan unos de otros y las comunidades se posicionen como contextos ricos y significativos para investigar" (Tolbert, 2015, p. 1327).

Se partió de la premisa de que la educación a lo largo de la historia de la humanidad ha estado vinculada con momentos históricos y visiones de sociedad permeadas por tensiones o intereses internos - nacionales - y externos - internacionales-. Se requiere, por lo tanto, pensar la pertinencia de una postura dialógica entre la escuela y su contexto cultural y ambiental para generar espacios de enseñanza y aprendizaje, en los cuales las subjetividades e intersubjetividades del profesorado y de los estudiantes se reconozcan en un marco de dignidad para construir a partir de ella sentidos y significados situados y relevantes en torno a la ciencia. Para ello, se debe tener presente que "la enseñanza y el aprendizaje se consideran constituyentes dialécticos teniendo en cuenta que su relación se caracteriza por la irreductibilidad y la copresencia, donde cada entidad presupone la existencia del otro" (Tobin, 2012, p. 3).

Así, la posibilidad de comprender las concepciones del profesorado de básica y media permitió ampliar la perspectiva de la educación en ciencias para la formación más allá de discursos en torno al capital humano, las competencias, el emprendimiento o la competitividad. Como lo mencionan Blázquez (2012) y Álvarez (2010), si nos ubicamos fuera del etnocentrismo económico y/o didáctico que solo considera la economía de las prácticas desde una visión economicista, los procesos sociales podrían reencontrar parte de su sentido y superar el eurocentrismo que ha guiado la educación desde el siglo XV, a partir de los principios historiográficos, universalistas, civilizatorios y progresistas.

Lo anterior se articula a la pertinencia de reconocer en Latinoamérica que la diversidad cultural de los pueblos debe ser considerada como un factor de equilibrio, mas no de división. La explotación de este potencial significa la adquisición de su propio destino y su fortalecimiento para su apertura al mundo, ya que se genera un empoderamiento que daría lugar a intercambios y no a exclusiones (Unesco, 2004).

\section{Marco teórico y planteamiento del problema}

La posibilidad de comprender las concepciones del profesorado sobre la educación en ciencias en cuatro contextos culturales y ambientales de Colombia invita a volver la mirada a la escuela, no solo como institución social, sino como escenario de construcción de sentido — desde lo subjetivo- y de significado — desde lo intersubjetivo-, a partir de posturas dialógicas que permiten: el reconocimiento del otro — sus realidades, sus miedos, sus imaginarios, sus experiencias-; la exploración de ideas o visiones del mundo 
expresadas por los sujetos; y la construcción de redes de conocimiento con el otro, a partir de procesos de análisis, síntesis e inferencia que permitan vincular argumentos que lleven a comparar, discernir, tomar postura y actuar sobre las formas de comprender e interactuar. De acuerdo con Mosquera y Rodríguez (2018), “una formación que se base únicamente en lo universal no crea una cultura escolar que favorezca forjar el sujeto" (p. 261), dado que esto requiere de las experiencias del sujeto en todo su recorrido vital, en relación con los otros y el mundo. Aquí, el contexto juega un papel fundamental, pues, con la orientación del docente, el estudiante puede hacer construcciones personales basadas en experiencias que vive en su cultura.

Por otra parte, Ramírez (2011) menciona que en "cada una de las culturas locales y subregionales se reivindican características propias que se derivan de la apropiación de un espacio particular, una experiencia histórica compartida y un sentido de pertenencia" (p. 153), lo cual se enlaza con la postura de Dentzau (2014), al plantear que "comprender el lugar implica reconocer que para el individuo es altamente situacional y tiene un significado específico que surge de sus identidades culturales y sociales únicas" (p. 166), por lo cual urge abordar críticamente los diálogos entre la ciencia con las prácticas culturales y ambientales, "inmersos en valores políticos y religiosos e insertos en contextos socio-históricos múltiples" (Astudillo \& Rivarosa, 2012, p. 18).

Desde esta perspectiva, cabe señalar que la cultura actúa como un fuerte agente de socialización que influye en el procesamiento de la información y la cognición (Joy \& Kolb, 2009), motivo por el cual se reconoce que, cuando se enseña, se está enseñando cultura dentro de un contexto ambiental, de manera que el conocimiento, las habilidades, las actitudes, junto con las disposiciones axiológicas y éticas, son todas manifestaciones de la cultura y las relaciones de la sociedad con la naturaleza, dado que no son de alguna manera inmunes a ellas. "Cuando se enseña, se está pasando no solo lo que sabemos, sino cómo lo conocemos, así como la base para aceptarlo como conocimiento útil, y los valores que representan" (Parrish \& Linder-VanBerschot, 2010, p. 5)

En consecuencia, generar reflexiones sobre la incidencia de los contextos culturales y ambientales permite reconocer el dilema al que se enfrenta el profesorado de ciencias sobre un territorio, que consiste en determinar cómo se reconoce a ese colectivo que disfruta de ciudadanía y culturalidad (Abad, 2014, p. 18). Este dilema implica el reto de explorar las preconcepciones, ideas y comportamientos intuitivos de los estudiantes que influyen en la construcción de los conocimientos científicos (Fernández et al., 2002). En este sentido, la relación de lo cultural y lo ambiental en la educación en ciencias permite profundizar la integración relevante del contexto, desde los aprendizajes situados y con sentido que pueden generarse con una ciencia escolar que vincule la esencia singular del entorno, que se teje entre lo social y lo natural para formar ciudadanos desde la diversidad y para la diversidad.

Así, pensar la educación en ciencias teniendo en cuenta los entramados de significados culturales y ambientales lleva a traspasar las fronteras del análisis psicológico o cognitivo del aprendizaje y de la enseñanza, ya que "los estudiantes y los profesores, al igual que el resto de las personas, poseen un conjunto de concepciones sobre el medio, en general, y sobre el medio escolar, en particular" (Porlán et. al., 1997 p. 156). Por otra parte, Smith y Gunstone (2009) plantean que en este momento se reconoce la importancia de ubicar a los individuos 
dentro de una comunidad. Es decir, no solo se debe ver a los demás como individuos, sino también como la comunidad que ha hecho que el individuo sea posible, lo cual se reitera desde Halliday (1982), quien dice que, mediante los actos cotidianos de significación, la gente representa la estructura social, al afirmar sus propias posiciones y sus propios papeles, así como al establecer y trasmitir los sistemas comunes de valor y conocimiento. A esto se suma el reconocimiento del otro, para acercar, comunicar y construir la dignidad humana desde lo individual y lo colectivo. Lo anterior se complementa con los postulados de Porta (2013) sobre los legados de Mijail Bajtín, en los que la posibilidad de un sí mismo, de una subjetividad, está condicionada por la mirada ajena, que no es otra que la mirada del mundo, la mirada del contexto, la mirada social. Allí los procesos de significación —-materiales y discursivos, a la vez como corporales y enunciativos- están estrechamente vinculados a las condiciones desde las que se realizan y al carácter local, situado e ideológico. Por ello, pensar una educación en ciencias sensible al contexto "implica prácticas pedagógicas comprometidas con la promoción del diálogo intercultural, es decir, entre la cultura de la ciencia y la cultura de los estudiantes" (Baptista, 2015, p. 586).

En consecuencia, esta investigación invita a pensar la educación en ciencias desde el reconocimiento de la diferencia y las oportunidades para la construcción dialógica del conocimiento científico escolar, con el ánimo de generar aportes en la disciplina de la didáctica de las ciencias, a partir de la formulación del siguiente problema: ¿cómo son las concepciones de profesores en ejercicio de básica y media sobre la educación en ciencias desde diferentes contextos culturales y ambientales de Colombia? Dicho problema se delimitó en los siguientes aspectos:

1. Pretendió generar procesos de comprensión sobre las concepciones del profesorado de básica y media sobre la educación en ciencias, a partir de la premisa de que las concepciones son creencias que implican una convicción o valoración sobre algo y de que en ellas juega un importante papel la viabilidad, el componente social y la predisposición para actuar (Mellado, 1996).

2. Se realizó a través de estudios de casos por medio de entrevistas semiestructuradas, observación directa en el aula y análisis documental de las construcciones que soportan el currículo.

3. Se buscó fortalecer pedagógica y didácticamente la educación en ciencias y potenciar la vinculación del contexto cultural y ambiental en la construcción del conocimiento científico escolar.

\section{Metodología}

Teniendo en cuenta que la investigación fue de carácter cualitativo y se ubicó en el paradigma interpretativo, ontológicamente abordó una realidad de naturaleza cultural que es diversa y - a nivel epistemológico- subjetiva, tanto por las construcciones de sentido de cada individuo como por las tramas de significado de su colectividad. En términos metodológicos, se situó en el ámbito hermenéutico. Cabe señalar que, si bien el proceso interpretativo puede presentar diferentes enfoques de abordaje en esta investigación, se hizo la aproximación desde el interaccionismo simbólico, el cual está orientado a reconocer cómo los seres humanos actúan respecto de las cosas según los significados que estas tienen para ellos (Álvarez-Gayou, 2003). 
Por otra parte, Yin (2002) plantea que un estudio de caso es una pregunta empírica que investiga un fenómeno contemporáneo dentro de su contexto de vida real, sobre todo cuando los límites entre el fenómeno y el contexto no son claramente evidentes. Esto se reitera desde los aportes de Álvarez y San Fabián (2012), cuando plantean que los estudios de caso presentan ciertas características que permiten diferenciarlos de otros métodos de investigación:

1. Realizan una descripción contextualizada del objeto de estudio.

2. Son estudios holísticos, dado que el investigador ha de tratar de observar la realidad con una visión profunda $\mathrm{y}$, asimismo, ha de tratar de ofrecer una visión total del fenómeno objeto de estudio, mientras refleja la complejidad de este.

3. Evidencian la peculiaridad y la particularidad de cada realidad/situación a través de una descripción densa y fiel del fenómeno investigado.

4. Los estudios de caso incorporan múltiples fuentes de datos. Su análisis se ha de realizar de modo global e interrelacionado.

La población de este estudio fue de ocho profesores y profesoras en ejercicio de ciencias naturales en educación básica y media ubicados así: dos en Leticia -Amazonas-, dos en La Estrella - Antioquia-, dos en Carmen de Bolívar - Bolívar- y dos en Bogotá. En esta investigación se desarrolló un diseño de casos múltiples, con el propósito de ubicar a cada individuo - en lo personal y en su entorno - para tener una comprensión global de la situación que se estudió (Ander-Egg, 2003). Asimismo, la investigación se desarrolló con rigor, credibilidad y confidencialidad en cuanto al tratamiento de los datos y a los resultados. Estos últimos provienen de la recolección de información a través de: entrevistas semiestructuradas - cuya estructura, avalada por expertos, se basó en seis dimensiones producto de la revisión teórica de la tesis doctoral: conceptual, metodológica, actitudinal, axiológica, ética y política-; la observación de aula con un curso en cada uno de los ocho casos -durante el último semestre de 2018-; y el análisis de los respectivos microcurrículos elaborados por los docentes. Cabe señalar que cada una de las entrevistas fue transcrita, codificada y analizada a partir de un proceso de triangulación con datos recolectados con las otras técnicas y bibliografía complementaria.

\section{Resultados}

La investigación permitió identificar experiencias y eventos donde se integra el contexto cultural y ambiental, lo cual permite reconocer cómo el profesorado se involucra y toma decisiones que vinculan las dimensiones conceptuales, metodológicas, actitudinales, axiológicas, éticas y políticas, que trascienden las reflexiones netamente asociadas a los contenidos o a las actividades a desarrollar en el aula.

De acuerdo con la revisión teórica y de las construcciones que fue realizando la investigadora a lo largo de la tesis doctoral, se reconoció que en la escuela confluyen diversas clases de conocimientos, ideas e incluso formas de ver y comprender en el mundo - cosmovisiones, ideas populares, referencias de la vida cotidiana-, constituidas por las experiencias, percepciones e interacciones, tanto del profesorado como de estudiantes, 
junto con las de otros sujetos a nivel familiar, comunitario o regional, que indudablemente entran en diálogo en la escuela, la cual está permeada por el momento histórico y social, como también por los contextos culturales y ambientales en el que está inmersa. Se logró identificar que, en las concepciones del profesorado, entran en juego "factores subjetivos, biográficos y experienciales, así como aspectos contextuales que forman un sistema idiosincrático de conocimientos, producto de la elaboración personal de sus ideas en un contexto institucional y social determinado" (Briceño \& Benarroch, 2012, p. 25).

Así, la educación en ciencias se puede comprender como el proceso que permite desarrollar escenarios de enseñanza y aprendizaje que potencien la configuración de redes de sentido - desde lo subjetivo- y significado - desde lo intersubjetivo- en el estudiantado. Allí se vincula lo individual y lo social del aprendizaje, lo cual permite construir relaciones entre el conocimiento científico escolar y el mundo de la vida, para que el estudiantado pueda configurar el conocimiento científico escolar. Este no solo implica conocimientos, habilidades, actitudes frente a las ciencias, sino también configuraciones sobre el ser en lo social, dado que las ciencias no son neutrales al estar influenciadas por la sociedad donde se desarrolla, en términos culturales, económicos y políticos. Como lo plantean Suriel y Atwater (2012), "los sistemas de conocimiento son dinámicos e influenciados cultural e históricamente, lo cual implica reconocer diversas cosmovisiones y formas de construcción y legitimación del conocimiento" (p. 1273).

En este sentido, es pertinente mencionar que las transformaciones subjetivas e intersubjetivas que se pueden generar en el aula influyen en la cultura del contexto inmediato o mediático - a través de las tecnologías - y están intercedidas por las acciones comunicativas, que a su vez implican el desarrollo de facultades de pensamiento para discernir, argumentar, debatir, proponer, elegir y corresponsabilizarse. Esto permite vislumbrar las conexiones interdependientes entre sujeto, colectividad, cultura y ambiente, que deben ser reconocidas en el ámbito escolar para generar diálogos entre ellas.

Así, el reconocimiento intencionado del contexto cultural y ambiental en la didáctica de las ciencias puede brindar más elementos al profesorado de ciencias para la formulación de relaciones en la ciencia escolar, a través de intercambios y escenarios dialógicos para identificar diferentes epistemologías alrededor de la ciencia y cómo la ciencia escolar puede comprenderse y construirse desde relaciones con el contexto local, nacional o global, de manera progresiva e incluyente entre sí.

A continuación, se describen dos de los cuarenta y cinco episodios identificados en los estudios de caso (tabla 1), que permiten reconocer cómo el profesorado genera relaciones entre la educación en ciencias y el contexto cultural y ambiental. A través de los dos episodios se logra identificar la relación con dos categorías y subcategorías emergentes del total de las construidas en la investigación (tabla 2).

El análisis hermenéutico efectuado con el programa Atlas.ti sobre las concepciones que manifestó el profesorado acerca de la educación en ciencias desde diferentes contextos culturales y ambientales permitió identificar las siguientes categorías, a partir de las cuales se pueden reconocer oportunidades para construir diseños curriculares y didácticos situados, con el reconocimiento del territorio y de la población, para tejer relaciones con sentido y significado que contribuyan al desarrollo de aprendizajes pertinentes y relevantes. 
Tabla 1

Episodios identificados en los estudios de caso

\begin{tabular}{|c|c|}
\hline \multicolumn{2}{|c|}{ Ubicación del establecimiento educativo } \\
\hline \multicolumn{2}{|c|}{ Bogotá } \\
\hline Categoría & Subcategoría \\
\hline Salud y bienestar & Promoción del bienestar \\
\hline \multicolumn{2}{|c|}{ Fragmento } \\
\hline $\begin{array}{l}\text { ¿Qué problemáticas medic } \\
\text { - Pues problemática aml } \\
\text { auditiva. Entonces el niñ } \\
\text { va arrastrando el puesto. } \\
\text { día una niña se estaba es } \\
\text { niña, en su casa su papá } \\
\text { y gritan. Y ella me decía } \\
\text { y ese bullicio, esa chiflac } \\
\text { respuesta de docente) }\end{array}$ & $\begin{array}{l}\text { lidad o en el barrio? } \\
\text { s trabajo mucho, por ejemplo, la contaminación } \\
\text { el brazo de un lugar a otro dentro del salón-y } \\
\text { grita, entonces es una normalización... El otro } \\
\text { ba la chifladera. Y después, preguntándole a la } \\
\text { mpo porque se pelean. Entonces gritan y gritan } \\
\text { ma para no escuchar. Entonces, claro, los gritos } \\
\text { lo que está viviendo en la casa. (Fragmento de }\end{array}$ \\
\hline
\end{tabular}

\begin{tabular}{l} 
Análisis \\
\hline La profesora reconoce el contexto cultural y ambiental en el cual está inmersa la escuela como un \\
entorno donde se presentan problemáticas que la han llevado a activar alarmas para generar redes \\
de apoyo asociadas con la atención a estudiantes, no solo dentro de la escuela, sino también a través \\
de instituciones de carácter distrital, lo cual permite inferir que las intencionalidades pedagógicas \\
y las construcciones didácticas del profesorado trascienden el aula de clase. Esto lleva a pensar la \\
educación desde una construcción de enlaces que pueden aportar a la atención de necesidades o \\
problemáticas del estudiantado, al desligar posturas de indiferencia. Por otro lado, se manifiestan \\
percepciones asociadas con temas de seguridad dentro de un espacio cercano a la escuela -un \\
caño-, el cual se vincula también con un evento que afectó a un estudiante cuando ella describe: \\
"un niño que tenía... hace como tres años, en noveno grado, la mamá era drogadicta y se la pasaba \\
en ese caño... seguramente alguien le dijo algo y no volvió, sencillamente no volvió y no se supo \\
nada más". Lo anterior permite entrever cómo ella se involucra, se sensibiliza y hace seguimiento \\
desde una dimensión política y ética de la situación. Desde otro punto de vista, también se observa \\
cómo la profesora destaca que el contexto ambiental presenta situaciones de contaminación por \\
residuos sólidos, que no solo afectan el curso del agua en el caño, sino que generan la presencia de \\
roedores y olores que directamente pueden llegar a afectar la escuela. En consecuencia, podría haber \\
problemáticas de salud pública en el sector. Así, enseñar y aprender ciencias es un proceso dialógico \\
que, además de realizarse entre docentes y estudiantes, está inmerso en contextos particulares y \\
complejos (Ruiz et al., 2015).
\end{tabular}
complejos (Ruiz et al., 2015).

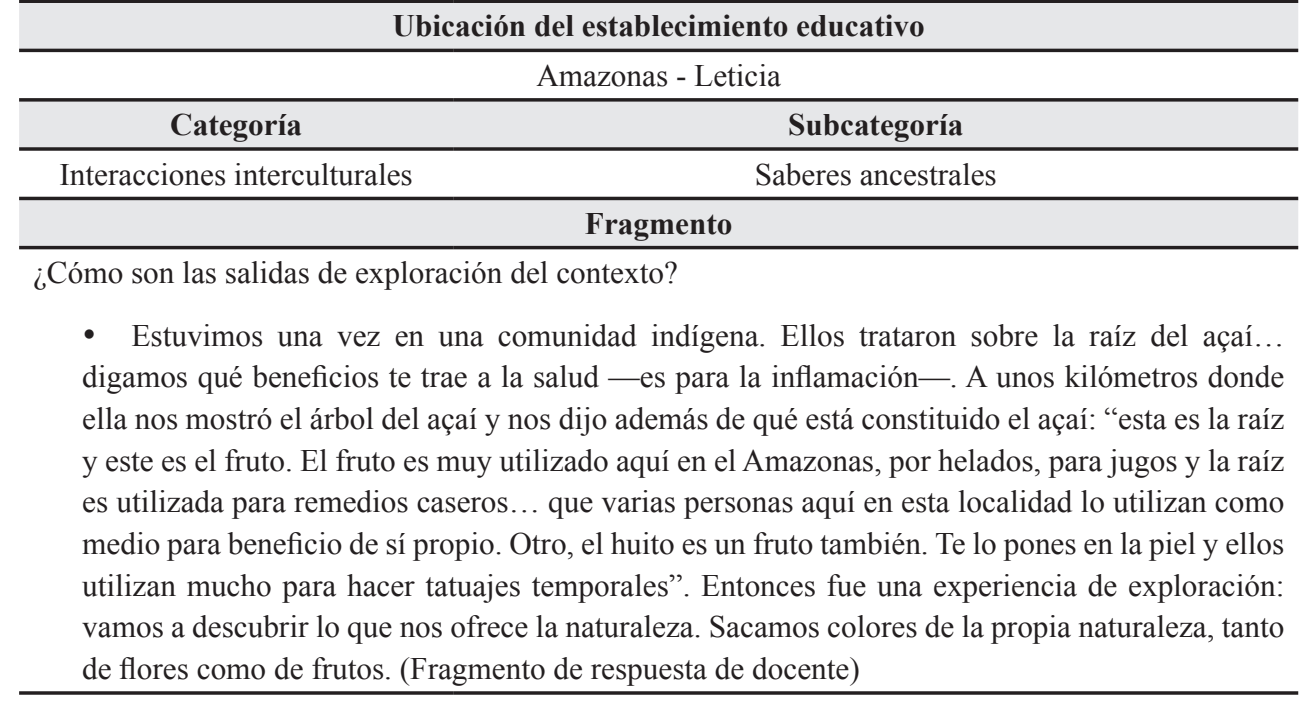


Análisis

En este fragmento se logra identificar la exploración de saberes tradicionales sobre el açaí de una comunidad indígena. Cuando la profesora relata que "el fruto es muy utilizado aquí en el Amazonas, por helados, por este... jugos y la raíz es utilizada para remedios caseros... que varias personas aquí en esta localidad lo utilizan como medios para beneficio de sí propio", permite entrever el reconocimiento de cosmovisiones culturales diferentes. Se evidencia la búsqueda de oportunidades para generar escenarios dialógicos con la pluralidad de identidades locales, de lo cual se puede inferir que, desde la ciencia escolar, se puede abordar el fomento de voluntades para convivir en comunidad y distinguir los legados culturales de los discursos científicos, al diferenciar los respectivos contextos de uso en un marco de respeto. En este sentido, se contribuye a la valoración y comprensión de las tradiciones o expresiones vivas de nuestros antepasados relacionadas con la naturaleza y su importancia como patrimonio inmaterial. Lo anterior se ratifica desde la Declaración Universal de la Unesco sobre la Diversidad Cultural del 2001, cuando menciona que la cultura adquiere formas diversas a través del tiempo y del espacio que hacen parte de los legados de la humanidad.

Tabla 2

Categorías y subcategorías emergentes a través de las cuales se puede integrar el contexto cultural y ambiental en la educación en ciencias

\begin{tabular}{cccc}
\hline Categoría & $\begin{array}{c}\text { Propiedades de } \\
\text { la categoría }\end{array}$ & Subcategorías & Propiedades de la subcategoría \\
\hline
\end{tabular}

\begin{tabular}{clcl} 
Interacciones & $\begin{array}{l}\text { En este escenario } \\
\text { se relacionan las } \\
\text { intercultu- } \\
\text { rales } \\
\text { bencias con sa- }\end{array}$ & $\begin{array}{l}\text { Diálogos intercul- } \\
\text { turales }\end{array}$ & $\begin{array}{l}\text { Aproximarse al otro, que significa un acer- } \\
\text { camiento al tema de la subjetividad y la } \\
\text { identidad cultural (Molina et al., 2014). }\end{array}$ \\
\cline { 3 - 4 } & $\begin{array}{l}\text { nes ancestrales o } \\
\text { epistemologías } \\
\text { de comunidades } \\
\text { étnicas. }\end{array}$ & $\begin{array}{c}\text { Saberes ances- } \\
\text { trales }\end{array}$ & $\begin{array}{l}\text { Si bien el conocimiento científico es una } \\
\text { forma específica de conocimiento, no se } \\
\text { acepta su sobrevaloración en detrimento de } \\
\text { otros conocimientos ni la discriminación de } \\
\text { estos últimos (Cobern \& Loving, 2001). }\end{array}$
\end{tabular}

Se debe ser consciente de que cada proyecto, cada obra o actividad que se desarrolle, ocasiona sobre el entorno en

Impacto de la

Relaciones de las ciencias con aspectos

Relaciones entre el mundo natural y el social ambientales y el impacto de las prácticas humanas en los ecosistemas, sostenibilidad y biodiversidad. actividad humana que se ubica un cambio, una modificación en las condiciones existentes. Si dicha modificación significa una perturbación, o un impacto negativo, esta debería ser minimizada con base en estudios de impacto ambiental (Dellavedova, 2011). Conservación de la biodiversidad y cómo restaurar, rehabilitar y recuperar Protección o ecosistemas, tanto para la conservación restauración como para asegurar la sostenibilidad y/o recuperación de servicios ecosistémicos (Armenteras \& Vargas, 2016).

Exploración/ aprovechamiento

responsable
Participación e integración de los sujetos en la vida cotidiana dentro del mundo cambiante, sin menoscabo de los procesos ambientales (Arias, 2016). 


\begin{tabular}{|c|c|c|c|}
\hline \multirow{3}{*}{$\begin{array}{l}\text { Entorno } \\
\text { familiar, } \\
\text { comunitario o } \\
\text { multimedial }\end{array}$} & \multirow{3}{*}{$\begin{array}{l}\text { Relaciones de } \\
\text { las ciencias } \\
\text { con aspectos } \\
\text { que vinculan } \\
\text { interacciones } \\
\text { en la familia, } \\
\text { en el barrio, } \\
\text { en la ciudad/ } \\
\text { municipio/ } \\
\text { vereda y con } \\
\text { contenidos } \\
\text { virtuales o } \\
\text { de medios de } \\
\text { comunicación }\end{array}$} & $\begin{array}{l}\text { Construcciones } \\
\text { culturales locales }\end{array}$ & $\begin{array}{l}\text { Dentro de la identidad cultural están las } \\
\text { diferentes construcciones de sentido que } \\
\text { sobre sí mismos producen colectivos de } \\
\text { personas, comunidades, que se transforman } \\
\text { históricamente (Altez, 2016). }\end{array}$ \\
\hline & & $\begin{array}{l}\text { Relaciones con } \\
\text { la familia y la } \\
\text { comunidad }\end{array}$ & $\begin{array}{l}\text { Formas de construir, fortalecer, cuidar } \\
\text { y sostener alianzas con la familia o el } \\
\text { entorno comunitario para romper la inercia } \\
\text { instaurada en la manera de entender las } \\
\text { relaciones dentro y fuera de los centros } \\
\text { educativos (Simón et al., 2016). }\end{array}$ \\
\hline & & $\begin{array}{l}\text { Fomento de } \\
\text { interacción con } \\
\text { recursos digitales } \\
\text { o multimediales }\end{array}$ & $\begin{array}{l}\text { Reflexión sobre el modo en que las } \\
\text { tecnologías configuran la significación, } \\
\text { percepción, representaciones e imaginarios, } \\
\text { al establecer nuevas tensiones en la } \\
\text { producción simbólica y cultural en la } \\
\text { escuela (Martín, 2016). }\end{array}$ \\
\hline & & $\begin{array}{l}\text { Gestión del } \\
\text { riesgo —físico, } \\
\text { psicológico, } \\
\text { emocional- }\end{array}$ & $\begin{array}{l}\text { Procesos o estrategias socioeducativas que } \\
\text { propendan por el desarrollo digno y sano, } \\
\text { para lograr una ciudadanía plena sin riesgos } \\
\text { de exclusión (Martínez et al., 2018). }\end{array}$ \\
\hline $\begin{array}{l}\text { Salud y } \\
\text { bienestar }\end{array}$ & $\begin{array}{l}\text { En este escenario } \\
\text { se vinculan } \\
\text { las ciencias } \\
\text { con aspectos } \\
\text { asociados a los } \\
\text { estados físicos, } \\
\text { mentales, } \\
\text { emocionales } \\
\text { y sociales, } \\
\text { tanto desde } \\
\text { lo individual } \\
\text { como desde lo } \\
\text { colectivo. }\end{array}$ & $\begin{array}{l}\text { Promoción del } \\
\text { bienestar }\end{array}$ & $\begin{array}{l}\text { En efecto, la noción de bienestar subjetivo } \\
\text { apela a distintos principios - desde la } \\
\text { Convención de los Derechos del Niño-, } \\
\text { tales como la no discriminación, al } \\
\text { considerar el bienestar subjetivo de los } \\
\text { grupos de niños, niñas y adolescentes, } \\
\text { y al reconocer sus particularidades y } \\
\text { diferencias según edad, género, etnia, etc. } \\
\text { Asimismo, persigue el cumplimiento del } \\
\text { interés superior del niño al definirlos como } \\
\text { ciudadanos y ciudadanas con derechos, } \\
\text { y al preocuparse por su supervivencia y } \\
\text { desarrollo mientras se promueve una visión } \\
\text { holística de la infancia y la adolescencia, } \\
\text { ya que se les otorga el mismo peso a los } \\
\text { derechos civiles, políticos, sociales y } \\
\text { económicos, de forma relacional, universal } \\
\text { e indivisible. Finalmente, garantiza el } \\
\text { derecho de niños, niñas y adolescentes } \\
\text { de dar su opinión, en relación con los } \\
\text { aspectos que consideran importantes para } \\
\text { su bienestar según su etapa de desarrollo } \\
\text { (Oyanedel et al., 2015) }\end{array}$ \\
\hline
\end{tabular}




\begin{tabular}{|c|c|c|c|}
\hline \multirow{2}{*}{$\begin{array}{l}\text { Responsabi- } \\
\text { lidad ética y } \\
\quad \text { social }\end{array}$} & \multirow{2}{*}{$\begin{array}{l}\text { Reflexiones } \\
\text { asociadas con } \\
\text { aspectos de segu- } \\
\text { ridad, protección } \\
\text { y defensa de la } \\
\text { vida y la digni- } \\
\text { dad humana. }\end{array}$} & $\begin{array}{l}\text { Ejercicio de dere- } \\
\text { chos y calidad de } \\
\text { vida }\end{array}$ & $\begin{array}{l}\text { Según Marí et al. (2016), la ciudadanía ple- } \\
\text { na sería la que haría efectivos el progresivo } \\
\text { cumplimiento de los Derechos Humanos y } \\
\text { la progresiva incorporación de lo que se ha } \\
\text { denominado derechos de segunda, tercera y } \\
\text { cuarta generación, que incluyen el desarro- } \\
\text { llo de la autonomía, la expresión de la indi- } \\
\text { vidualidad, la perspectiva social, la partici- } \\
\text { pación social en todas sus dimensiones y la } \\
\text { inserción en proyectos comunes. }\end{array}$ \\
\hline & & $\begin{array}{l}\text { Asuntos de co- } \\
\text { rresponsabilidad } \\
\text { ciudadana }\end{array}$ & $\begin{array}{l}\text { Al acercarse a lo problemático y contradic- } \\
\text { torio de las sociedades, es pertinente ubicar } \\
\text { los conocimientos en la propia experiencia } \\
\text { cotidiana, a partir de tres grandes líneas: el } \\
\text { conocimiento del mundo y de las socieda- } \\
\text { des, el desarrollo del sentido de lo común y } \\
\text { lo público y el aprendizaje del marco ético } \\
\text { y normativo de convivencia (Marí et al., } \\
\text { 2016). }\end{array}$ \\
\hline \multirow[t]{2}{*}{ Innovación } & \multirow{2}{*}{$\begin{array}{l}\text { Experiencias que } \\
\text { generan sinergias } \\
\text { entre las ciencias } \\
\text { con iniciativas } \\
\text { que conducen al } \\
\text { diseño de prototi- } \\
\text { pos o estrategias } \\
\text { de intervención. }\end{array}$} & $\begin{array}{l}\text { Proyectos } \\
\text { productivos }\end{array}$ & $\begin{array}{l}\text { La actitud emprendedora se hace evidente } \\
\text { cuando los estudiantes piensan de manera } \\
\text { flexible, desarrollan la creatividad en la } \\
\text { solución de problemas, transforman acon- } \\
\text { tecimientos e identifican oportunidades } \\
\text { del entorno para planear, ejecutar, evaluar } \\
\text { y sistematizar sus actividades y proyectos } \\
\text { (Cifuentes \& Rico, 2016). }\end{array}$ \\
\hline & & $\begin{array}{l}\text { Construcción de } \\
\text { prototipos }\end{array}$ & $\begin{array}{l}\text { Según Tecpan y Hernández (2017), no basta } \\
\text { con que el prototipo funcione. Es necesario } \\
\text { que se demuestre la capacidad de explicar } \\
\text { los fenómenos presentes y se emplee para } \\
\text { ello lenguaje científico. }\end{array}$ \\
\hline
\end{tabular}

A través de estas categorías emergentes, se identificaron las co-ocurrencias de cada una de ellas con el programa Atlas.ti, las cuales se presentan en la gráfica 1.

Si bien la entrevista semiestructurada fue igual para los ocho docentes participantes, en el análisis categorial de las dos profesoras de Leticia se destacaron particularmente concepciones alrededor del desarrollo de diálogos interculturales, exploración de saberes ancestrales y la promoción de relaciones del mundo natural con el social desde la exploración/ aprovechamiento responsable, lo que despierta en el estudiantado la curiosidad y el sentido de pertenencia con su contexto y sus legados culturales.

En los dos casos del municipio de La Estrella, se vincularon experiencias de inclusión, la integración de aspectos asociados a la naturaleza de las ciencias y el fomento de interacciones con recursos digitales o multimediales, a partir del acompañamiento de la familia o de los cuidadores para orientar la exploración asertiva y generar ambientes de seguridad a la integridad física y psicológica de los niños y niñas en la red.

Por otra parte, con los dos profesores de Carmen de Bolívar, se destacaron particularmente concepciones alrededor de: el fomento de vínculos con el entorno familiar y comunitario; la importancia de reconocer las construcciones culturales locales para establecer puentes con el lenguaje y los conocimientos científicos; y de las relaciones del mundo natural y el 
social junto con la vinculación del contexto en propuestas de innovación desde proyectos productivos o sostenibles - como construcción de biodigestores con apoyo de la comunidad y las familias de los estudiantes-.

Finalmente, en el caso de Bogotá, se destacó la lectura del entorno como parte del rol docente para situar la ciencia escolar en asuntos de corresponsabilidad ciudadana, gestión del riesgo físico, psicológico o emocional y promoción del bienestar.

\section{Gráfica 1}

Co-ocurrencias que se identificaron de las categorías y subcategorías emergentes

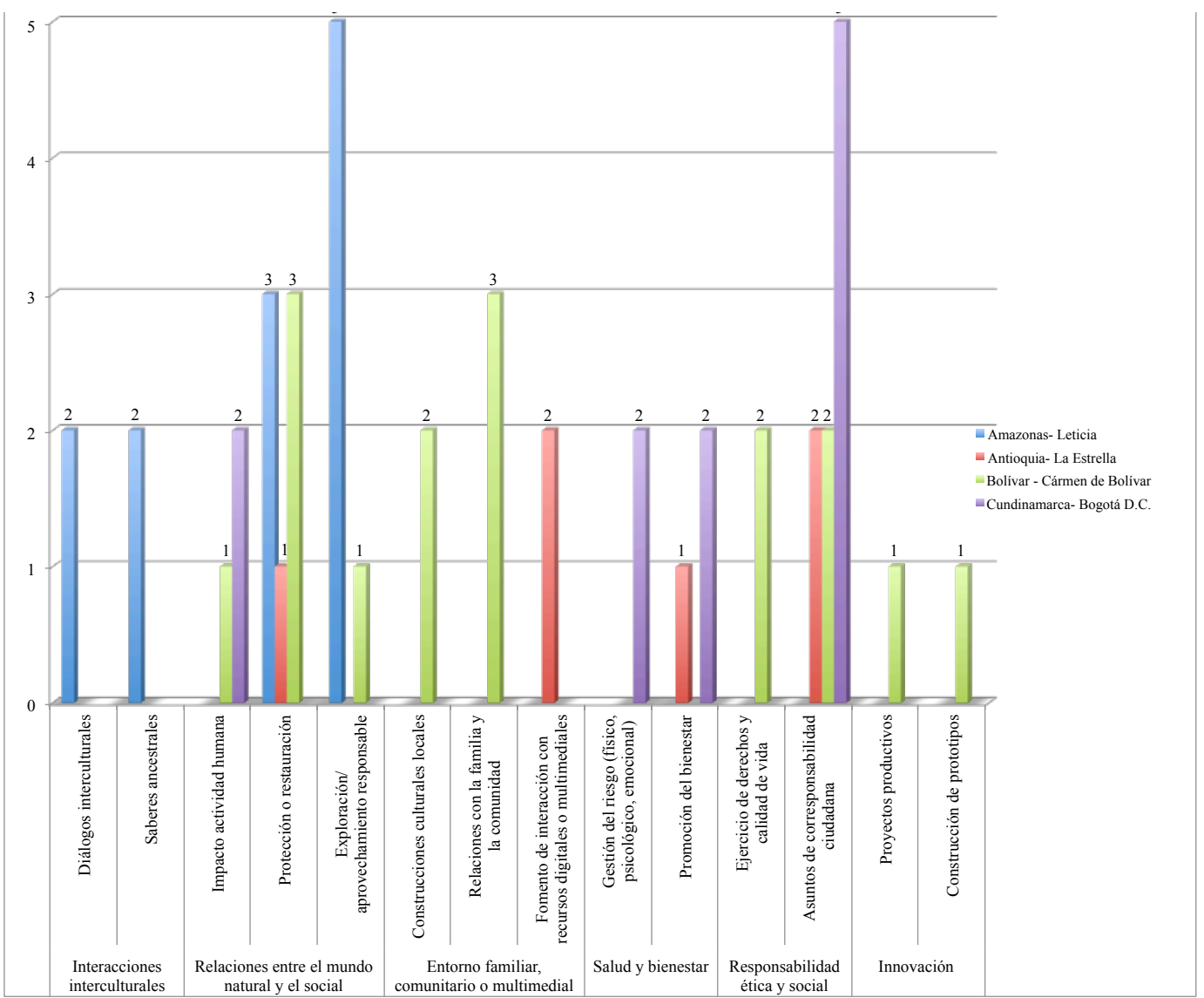

Las categorías emergentes y sus co-ocurrencias identificadas en el análisis de resultados permiten construir reflexiones didácticas y curriculares sobre la pertinencia de vincular el contexto y el enfoque diferencial para promover con los estudiantes aprendizajes situados, que brinden sentido y significado a los conocimientos desarrollados en la ciencia escolar.

En definitiva, un aspecto fundamental para despertar el interés y la motivación de los educandos es emplear diversas estrategias didácticas que les permitan recrear el trabajo de los científicos para que se sientan atraídos e interesados por el estudio y la comprensión de los fenómenos que los rodean, para así comenzar a desarrollar y a fortalecer los conocimientos conceptuales, procedimentales, actitudinales y las competencias científicas (Espinosa et al., 2016).

Asimismo, como lo referencia García (2015), no basta con destacar el papel "mediacional" del educador - lo que ya se ha hecho con bastante frecuencia en la investigación pedagógica一, 
sino que se debe considerar de manera integrada al aprendiz y al educador, es decir, plantear las propuestas curriculares a través de las características y las posibilidades que se abren en el aula. De este modo, la educación científica se abre como un proceso de construcción cultural en el que todo sujeto puede recibir, conocer y comprender información para tomar decisiones de forma crítica y reflexiva, al considerar aspectos éticos y estéticos, que suponen también el abordaje antropológico, sociológico e ideológico del proceso formativo (Arias \& Navarro, 2017).

\section{Conclusiones}

Esta investigación permitió establecer que el concepto de contexto cultural y ambiental es aquel construido por los sujetos a partir de los entramados de significados configurados a lo largo de su historia. Puede tener rasgos propios de la idiosincrasia local y estar influenciado por las condiciones medioambientales del entorno o por los aspectos sociales en las que ellos están inmersos cotidianamente, lo cual contribuye a generar trascendencias y/o transformaciones sobre las maneras de comprender e interactuar en el mundo a través del lenguaje. Este último permite generar escenarios didácticos dialógicos que influencian los procesos de educación en ciencias de los niños, niñas y jóvenes como sujetos políticos, es decir, como ciudadanos.

Esto implica rediseñar sistemáticamente el currículo, como lo formulan Fonseca y Gamboa (2017), a partir de situaciones problemáticas que respondan a necesidades sociales, donde estén implicados valores que sean objeto de debate y que tengan interés para la vida personal y comunitaria. Rodríguez (2017) reitera lo anterior cuando describe que el ser humano debe tender más hacia la humanización, mientras la ejerce con acciones concretas. En las instituciones educativas, esta utopía debe llevarse a una realidad subjetivada de saberes, vivencias, cotidianidades, estéticas y valores.

A partir de lo anterior, se concibe el currículo, desde los resultados de la investigación, como una construcción pedagógica y didáctica intencionada, que permite integrar los propósitos de educación de la sociedad —que están en sintonía con los referentes internacionales-, pero, al mismo tiempo, permite articular y potenciar la diversidad local, regional y nacional, para propender por el aprendizaje y el desarrollo de conocimientos de los estudiantes. Con esto se contribuye a trascender y transformar la cultura desde la deconstrucción y construcción de redes de sentidos y significados que permiten situar social, histórica, política, estética e incluso éticamente las ciencias, para configurar desde ellas una visión dignificante del ser humano, tanto en su dimensión individual como colectiva.

Bajo este escenario, el currículo debe contemplar que la función que desempeña el docente, el rol del estudiante y la implementación de las estrategias didácticas son trascendentales para construir ambientes de aprendizaje, que se enriquecen con las acciones y el conocimiento que cada uno aporta (Espinosa et al., 2016).

En conclusión, la relevancia de analizar las relaciones entre el contexto cultural y ambiental y la educación en ciencias se sustenta en la posibilidad de proponer diseños didácticos y potenciar facultades intelectuales, experimentales, actitudinales, culturales $\mathrm{y}$ sociales en los estudiantes, para que ellos identifiquen situaciones, necesidades o problemáticas interrelacionadas en sus territorios y reconozcan diferentes niveles de injerencia, exploración y abordaje de las ciencias y su posible influencia en la cultura y la 
sociedad. Lo anterior se logra al indagar diversas fuentes de información para analizar la fiabilidad de las referencias consultadas sobre las ciencias; al participar en procesos de análisis, comunicación y discernimiento según las sinergias entre ciencia convencional —erudita-, la ciencia escolar y el mundo de la vida; al desarrollar procesos de exploración y experimentación a partir de la comprensión de técnicas y procedimientos -en escenarios individuales o de trabajo en equipo-; al proponer alternativas o estrategias para dar solución o mitigar problemáticas que vinculan la ciencia, con la comunidad y la sociedad; y al tener en cuenta la postura personal y la de otros y generar estructuras argumentativas para comunicar y socializar los resultados.

Esto permite sintetizar el sentido que le otorgó el profesorado de básica y media a la vinculación del contexto cultural y ambiental en la educación en ciencias, a partir de sus predisposiciones para actuar, de sus valoraciones y de las relaciones que establecen con el componente social.

\section{Referencias}

Abad, D. (2014). Organización curricular de las identidades colectivas en España. Revista de Educación, 366, 12-42.

Altez, Y. (2016). Hermenéutica y configuración histórica de identidades culturales. Estudios sobre las Culturas Contemporáneas, 22(44). https://www.redalyc.org/ jatsRepo/316/31648715005/html/index.html

Álvarez, A. (2010). Formación de nación y educación. Siglo del Hombre Editores.

Álvarez, C., \& San Fabián, J. (2012). La elección del estudio de caso en investigación educativa. Revista Gazeta de Antropología, 28(1), 1-13. http://hdl.handle.net/10481/20644

Álvarez-Gayou, J. (2003). Cómo hacer investigación cualitativa: fundamentos y metodología. Paidós.

Ander-Egg, E. (2003). Métodos y técnicas de investigación social. Vol. IV. Grupo Editorial Lumen.

Arias, B. (2016). El consumo responsable: educar para la sostenibilidad ambiental. Aibi, Revista de Investigación, Administración e Ingeniería, 4(1), 29-34.

Arias, M., \& Navarro, M. (2017). Epistemología, ciencia y educación científica: premisas, cuestionamientos y reflexiones para pensar la cultura científica. Revista Actualidades Investigativas en Educación, 17(3), 1-20.

Armenteras, D., \& Vargas, O. (2016). Patrones del paisaje y escenarios de restauración: acercando escalas. Acta Biológica Colombiana, 21(1), 229-239.

Astudillo, C., \& Rivarosa, A. (2012). Un papel para la epistemología en la enseñanza de las ciencias. Revista Ciencia Escolar: Enseñanza y Modelización, 2(2), 11-34.

Baptista, G. (2015). Um enfoque etnobiológico na formação do professor de ciências sensível à diversidade cultural: estudo de caso. Ciência Educação, Bauru, 21(3), 585-603.

Blázquez, G. (2012). Los actos escolares: la invención de la patria en la escuela. Miño y Dávila 
Editores.

Briceño, J., \& Benarroch A. (2012). Concepciones y creencias sobre ciencia, aprendizaje y enseñanza de profesores universitarios de ciencias. Revista Electrónica de Investigación en Educación en Ciencia, 8(1), 24-41.

Cifuentes, J., \& Rico, S. (2016). Proyectos educativos productivos y emprendimiento en la juventud rural. Zona Próxima, Revista del Instituto de Estudios en Educación y del Instituto de Idiomas Universidad del Norte, (25), 87-102.

Cobern, W., \& Loving, C. (2001). Defining "Science" in a Multicultural World: Implications for Science Education. Science Education, 85, 50-67.

Dellavedova, M. (2011). Guía metodológica para la elaboración de una evaluación de impacto ambiental. Universidad de la Plata.

Dentzau, M. (2014). The value of place. Culture Studies of Science Education, 9, 165-171.

Espinosa, E., González, K., \& Hernández, L. (2016). Las prácticas de laboratorio: una estrategia didáctica en la construcción de conocimiento científico escolar. Entramado, 12(1), 266-281.

Fernández, I., Gil, D., Carrascosa, J., Cachapuz, A., \& Praia, J. (2002). Visiones deformadas de la ciencia transmitidas por la enseñanza. Revista Enseñanza de las Ciencias, 20(3), 477-488.

Fonseca, J., \& Gamboa, M. (2017). Aspectos teóricos sobre el diseño curricular y sus particularidades en las ciencias. Revista Redipe, 6(3), 83-112.

García, E. (2015). ¿Es posible una didáctica de la educación ambiental? Hacia un modelo didáctico basado en las perspectivas constructivista, compleja y crítica. Revista Eletrônica do Mestrado em Educação, volumen especial, 4-30.

Halliday, M. (1982). El lenguaje como semiótica social. Interpretación social del lenguaje y del significado. Fondo de Cultura Económica.

Joy, S., \& Kolb, D. (2009). Are there cultural differences in learning style? International Journal of Intercultural Relations, 33, 69-85.

Marí, R., Moreno, R., \& Hipólito, N. (2016). Educación y ciudadanía. Propuestas educativas desde la controversia. Foro de Educación, 14(20), 49-69.

Martín, M. (2016).La escuela frente a las nuev@s formas de leer, escribir y publicar. Catalejos, Revista sobre Lectura, Formación de Lectores y Literatura para Niños, 2(3), 84-101.

Martínez, B., Martínez, I., \& Alonso, M. (2018). Colaboración y redes socioeducativas para la inclusión de la infancia vulnerable. Education Siglo XXI, 36(2), 21-42.

Mellado, V. (1996). Concepciones y prácticas de aula de profesores de ciencias en formación inicial de primaria y secundaria. Revista Enseñanza de las Ciencias, 14(3), 289-302.

Molina, A., Mosquera, C., Utges, G., Mojica, L., Cifuentes, M., Reyes, J., Martínez, C., \& Pedreros, R. (2014). Concepciones de los profesores sobre el fenómeno de la diversidad cultural y sus implicaciones en la enseñanza de las ciencias. Universidad Distrital Francisco José de Caldas. 
Mosquera, C., \& Rodríguez, M. (2018). Proyecto educativo como fundamento para pensar la subjetividad política desde la cultura escolar. El Ágora USB, 18(1), 255-267. http:// dx.doi.org/10.21500/16578031.2771

Oyanedel, J., Alfaro, J., \& Mella, C. (2015). Bienestar subjetivo y calidad de vida en la infancia en Chile. Revista Latinoamericana de Ciencias Sociales, Niñez y Juventud, 13(1), 313-327.

Parrish, P., \& Linder-VanBerschot, J. (2010). Cultural Dimensions of Learning: Addressing the Challenges of Multicultural Instruction. International Review of Research in Open and Distance Learning, 11(2), 1-19.

Porlan R., Rivero A., \& Martín, R. (1997) Conocimiento profesional y epistemológica de los profesores I: teoría, métodos e instrumentos. Revista enseñanza de las ciencias, 15 (2), 155-171.

Porta da, E. (2013). Pensar las subjetividades contemporáneas: algunas contribuciones de Mijaíl Bajtín. Estudios semióticos, 9(1), 47-54.

Ramírez, R. (2011). Tendencias de la historia regional en Colombia. Problemas y perspectivas recientes. Historelo, Revista de Historia Regional y Local, 3(5), 147-168.

Rodríguez, M. (2017). Currículum, educación y cultura en la formación docente del siglo XXI desde la complejidad. Revista Educación y Humanismo, 19(33), 425-440.

Ruiz, F., Tamayo, O., \& Márquez, C. (2015). La argumentación en clase de ciencias, un modelo para su enseñanza. E ducação e Pesquisa, 41(3), 629-646. https://doi.org/10.1590/ S1517-9702201507129480

Simón, C., Giné, C., \& Echeita, G. (2016). Escuela, familia y comunidad: construyendo alianzas para promover la inclusión. Revista Latinoamericana de Educación Inclusiva, $10(1), 25-42$.

Smith, D., \& Gunstone, R. (2009). Science Curriculum in the Market Liberal Society of the Twenty-first Century: 'Re-visioning' the Idea of Science for All. Science Education, 39, $1-16$.

Suriel, R., \& Atwater, M. (2012). From the Contribution to the Action Approach: White Teachers' Experiences Influencing the Development of Multicultural Science Curricula. Journal of Research in Science Teaching, 49(10), 1271-1295.

Tecpan, S., \& Hernández, C. (2017). Aprendizaje por indagación para la construcción de arquetipos en física; el caso de un curso para formación de profesores en Chile. Latin-American Journal of Physics Education, 11(2). https://dialnet.unirioja.es/servlet/ articulo? codigo $=6353439$

Tobin, K. (2012). Sociocultural Perspectives on Science Education. Second International Handbook of Science Education. Vol. 24. Springer.

Tolbert, S. (2015). "Because They Want to Teach You About Their Culture": Analyzing Effective Mentoring Conversations Between Culturally Responsible Mentors and Secondary Science Teachers of Indigenous Students in Mainstream Schools. Journal of Research in Science Teaching, 52(10), 1325-1361. 
Unesco (2004). L'UNESCO et la question de la diversite culturelle.

Unesco (2001). Declaración Universal de la Unesco sobre la diversidad cultural. $31^{a}$ reunión de la Conferencia General de la UNESCO.

Yin, R. (2002). Investigación sobre estudio de casos: diseño y métodos. Applied Social Research Methods Series. Vol 5. Sage Publications. 